\title{
Relevance Preserving Projection and Ranking for Web Image Search Reranking With Hierarchical Topic Awareness
}

\author{
Pallavi Wathore $^{1}$, P. B. Bhalerao ${ }^{2}$ \\ ${ }^{1}$ Department of Computer Science and Engineering Marthawada Shikshan Prasark Mandal's Deogiri Institute of Engineering \& \\ Management Studies, Aurangabad Maharashtra state, India 2015-16 \\ ${ }^{2}$ Assistant Professor Department of Computer Science and Engineering Marthawada Shikshan Prasark Mandal's Deogiri Institute \\ of Engineering \& Management Studies, Aurangabad Maharashtra state, India 2015-16
}

\begin{abstract}
Image Search re-ranking is as an effective way to improve the retrieval precision. Image search re-ranking has recently been proposed to refine image search results obtained from text-based image search engines. Most of the traditional re-ranking methods cannot capture both relevance and diversity of the search results at the same time. Or they ignore the hierarchical topic structure of search result. Each topic is treated equally and for certain queries are naturally in hierarchical organization, rather than simple parallel relation. Image Search Reranking (ISR) technique aims at refining text-based search results by mining images' visual content. Feature extraction and ranking function design are two key steps in ISR. Here a new re-ranking method "Topic-Aware Reranking (TARerank)" is proposed. TARerank describes the hierarchical topic structure of search results in one model, and seamlessly captures both relevance and diversity of the image search results simultaneously. By set of carefully designed features, through a structured learning framework, relevance and diversified images are modeled in TARerank, and then the model is learned from human- labeled training sample. The learned model is expected to predict re-ranking results with high relevance and diversity for testing queries. We collect an image search dataset and conduct the comparison experiments on it to verify the effectiveness of the proposed method. The experimental results demonstrate that the proposed TARerank outperforms the existing relevance-based and diversified re-ranking methods. Extensive experimental results on real-world datasets show that the proposed algorithms are effective. Moreover, the fact that only relevant images are required to be labeled makes it has a strong practical significance.
\end{abstract}

Keywords: Topic-Aware Re-ranking (TARerank), Image Search Reranking (ISR), Text Based Image Retrieval (TBIR), Topic Coverage (TC), Content Based Image Retrieval (CBIR)

\section{Introduction}

The exponential growth of the Web and the sheer amount of Web pages suggest that users are becoming more and more dependent on the search engines' ranking methods to discover information relevant to their needs. Typically, user expect to find such information in the top-ranked results, and more often than not they only look at the document snippets in the first few result pages and then they give up or reformulate the query. This can introduce a significant bias to their information finding process and calls for ranking methods that take into account not only the overall page quality and relevance to the query, but also the match with the users' real search intent when they formulate the query. Generally, most of the images stored on the Web have lots of tags added with user's subjective judgments not by the importance of them. So, in tagged Web image retrieval, they have become the cause of precision rate decrease on simple matching of tags to a given query. The existing web image search engines, including Bing, Google, and Yahoo!, retrieve and rank images mostly based on the visit count of the images. Image redundancy is still a problem area concerned. The ranking system provided is not the exact search result because rank is based on visit count alone once person open the image if it even irrelevant visit count get incremented. Nowadays, typical Image search system includes Search Reranking one as well as Text Based Image Retrieval (TBIR) module [11]. This lies in two facts: 1) TBIR suffers the problem of mismatching Image and its associate text and always neglect visual content; 2) Since TBIR has achieved limited success, Image Search Reranking is effective in improving the initial TBIR performance. ISR has been receiving great attention in recent TBIR [11][17][22]. The refinement of search result by employing image visual information to reorder the initial text based image search results is known as Image Search Reranking. If observed, the noisy text based image search results still contain satisfactory image in top hundreds of search results. Therefore, reordering these top ones with visual cues is possible to satisfy user's search experience with both accuracy and response time [11].

Two important aspects of current ISR approach is feature extraction and ranking function. Visual features always play a fundamental role in ISR and other image search applications [8][12][13][14], where lots of approaches have been employed and developed. Feature extraction strategies for Image Search Reranking (ISR) treat the first searched images as, two or more than two relevance degrees, e.g., pertinent, reasonable and irrelevant. It observed that the important samples match well with their inquiries, while unessential cases don't coordinate their questions. For a particular inquiry, every applicable image shares a comparable idea while each unimportant image ordinarily changes with distinctive ideas. Subsequently, lack of awareness of the contrast between diverse relevance degree gatherings can't guarantee the fitting visual representations, and debases the 


\section{International Journal of Science and Research (IJSR) \\ ISSN (Online): 2319-7064}

Index Copernicus Value (2013): 6.14 | Impact Factor (2014): 5.611

last ISR execution. On account of positioning capacity plan, the techniques additionally have a few confinements. For instance, graph based techniques [9][16] are computationally costly in diagram development and also positioning calculation, which restrains its application to substantial scale datasets. Web image search motors, e.g., Bing, Google, and Yahoo!, are executed by searching and indexing printed data connected with images, in spite of the fact that for huge scale image accumulations, content based image search is powerful. It experiences the downside that printed data can't extensively and considerably portray the rich substance of images. As an outcome, some insignificant images are seen in the search results. To handle the troubles in content based image search, visual image search re-ranking has been effective to join visual data of images to refine the content based search results.

\section{Literature Survey}

Image search plays an important role in day to day life. To improve the image search performance from various aspects, considerable research efforts have been made. To overcome "Curse of dimensionality" phenomenon, brought by highdimensional visual features, feature embedding methods are required [5]. Therefore methods such as Principal Component Analysis (PCA) [7], Linear Discriminative Analysis (LDA) [3], Local Preserve Projection (LPP) [4], and Marginal Fisher Analysis (MFA)[18] are used. Image Search Reranking has great research interest in recent years. It categorized into classification- based method, graph based method and learning to rank based method. Classification based method consist of classifier with train data directly from the initially search result then reorder all the documents by the relevance scores predicted from the classifier. The employed factor is Support Vector Machine (SVM) [10]. But it seems poorer result than other two methods, as it took it as simply classification problem and neglects their differences. Graph based method are implemented from random walk or Bayesian perspective. Consider Hsu et. Al [6] consist of reranking process as random walk over context graph, where nodes are represent document in initial search and edges between them are weighted by multimodal contextual similarities. To compute the final reranking result, the stationary probability of the random walk is used. From the probabilistic perspective Tian et al. [16] investigated the ranking problem and derived optimal reranking function. In that, textual information is modeled to reflect the disagreement between initial search result and reranking result, and visual information is modeled as a conditional prior to indicate the ranking scores consistency among visually similar samples. Recently for Image Search Reranking, learning to rank based method are promising technique. For example, Yang et al. [9] used two approaches for ranking function by learning the co- occurrence pattern between the target semantics and feature extracted from initial search list. Geng et al [2] represent Content based ranking model with large marginal structured output learning techniques, in which both textual and visual information are simultaneously leveraged in the ranking learning process.
Survey on Web Image Search Reranking techniques consists of various methods for efficient image search reranking such as:

\section{i) Relevance Feedback in retrieval of image}

In [1] Content Based Image Retrieval (CBIR) widely uses Relevance Feedback scheme which are based on SVM. From a huge database, retrieval of image record is challenging part, with fast increase of computer power. The key idea behind this work is taking the concept of classifier learning, as each classifier has its capability and property to classify the relevant and irrelevant image samples. To improve recognition performance classifier learning scheme contain group of week classifier images to work well. For effective SVM performance, the work uses random subspace method which shows more effectiveness. Most of the CBIR method worked in the same way. In the database a feature vector is extracted from each image and set of all feature vectors is organized as database index. At query time, a feature vector is extracted from the query image and it then matched with the feature vector in the index. The differences between various systems lie in the feature that they extract and in the algorithm that are used to compare feature vectors. The block diagram of basic CBIR system is as follows:

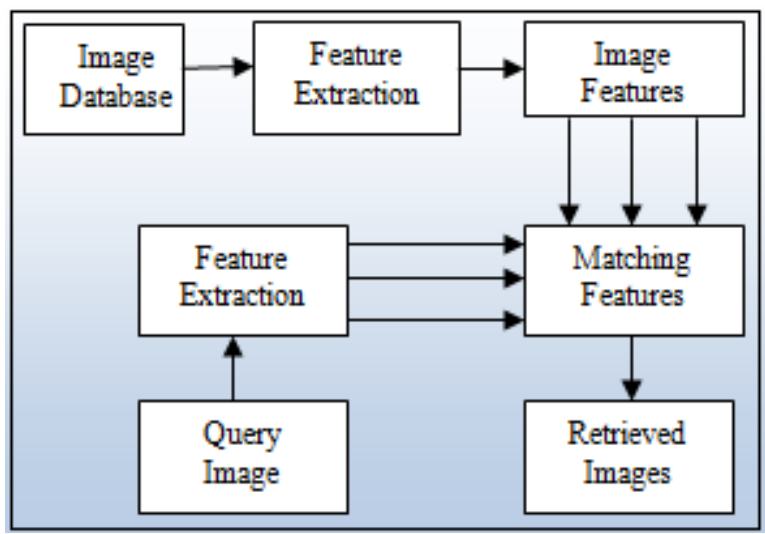

Figure 1: Block Diagram of CBIR

\section{ii) Attribute Feedback for Image Search}

In [23] Cai et. al presented a novel form of feedback for image search using relative attributes. In this method, several classifiers for all the pre-defined attributes feature and each image is represented by attribute feature consist of the responses from these classifiers. Presented method hypergraph is then used to model relationship between images by integrating attribute features and low level features. The Visual attribute joint hypergraph learning approach to explore the information. To model the relationship of all images, a hypergraph is constructed.

\section{iii) Bayesian Visual Re-ranking}

In [16] X. Tian et.al represented Bayesian Visual Reranking which model textual and visual information. It makes visual re-ranking as an optimization system in the Bayesian framework. In this scheme, textual information is replicated to reproduce divergence between texted based search result and re- ranked result which described as ranking distance. The visual information is replicated as a conditional prior to point visual consistency. By maximizing visual uniformity with minimizing distance of ranking, Bayesian Visual 


\section{International Journal of Science and Research (IJSR) \\ ISSN (Online): 2319-7064 \\ Index Copernicus Value (2013): 6.14 | Impact Factor (2014): 5.611}

reranking method obtains the best reranking consequences. Also a novel pair-wise method is used to figure out ranking distance more specifically. For visual uniformity, three different regularizes are investigated to extract the greatest way for its modeling.

\section{Proposed System}

The target of relevance-based re-ranking is to augment the relevance of the returned image list through reordering. However, main goal is to boost the relevance of everything in the rundown. The rundown tends of the subsequent positioning is to give back a substantial number of excess images that pass on tedious data. For example, visually comparative images have a tendency to show a highest priority on the rundown. As in [21], user leans towards the search result comprising of images that covered wide topics as well as in highly significant manner. To permit search result and pass on more data by expanding the topic scope (TC), enhanced re-ranking is proposed. Current re-ranking methods enhance the differing qualities, they experienced two difficulties. To begin with both relevance and assorted qualities are considered. To amplify relevance and diversified differences two stage enhancement can accomplished joint deal. Firstly, directing relevance based reranking to promote relevance and then enhancing TC by differentiate relevance based re-ranking result. Second, the expanded re-ranking topic assorted qualities by the low level visual features [19] which are not observed on the semantic differing qualities as of the semantic crevice.

Both relevance and broadened re-ranking doesn't catch the progressive TC structure of search result very well. More often they treat the topic as autonomously but the distinctive topics have diverse level of significance. More imperative topic wants to cover on uncommon topic. Additionally, it can manage most straightforward circumstances where all topics are free to manage with one another. In genuine applications, image returned from a certain query are normally in various level association, instead of delivering in straightforward parallel implementation. For instance, the query "Apple" incorporates two fundamental classification, "Organic product Apple" and result of "Apple Organization". In the topic natural organic product Apple, it further deals with subtopics, such as apple trees, red apple, crusty fruit-filled treat, apple juice and so forth.

Above problem can be solved out by the proposed re-ranking strategy, termed it as TARerank. At the point when certain query is submitted to the content based image search method, search result is returned which may contain some insignificant or copied image. TARerank reorder those images which acquire more palatable result which comprises of assorted images. TARerank can support various leveled TC structure of initial text based search result in one model and consistently catches both relevance as well as assorted qualities in image search at the same time. TARerank takes model in coverage area from preparation set by together advancing relevance and diversified differing qualities. Feature arrangement is initially removed to portray the relevance and assorted quality properties of self-assertive positioning result. At that point, a re-ranking model is found out to catch the reliance between the low-level features and the semantic-level TC and relevance. When the model is found out, we can utilize it to anticipate a re-ranking result which comprises of highly important images covering wide topics for another question. With a specific end goal to catch the various leveled topic structure, another basis, called normalized cumulated topic coverage (NCTC), is likewise proposed.

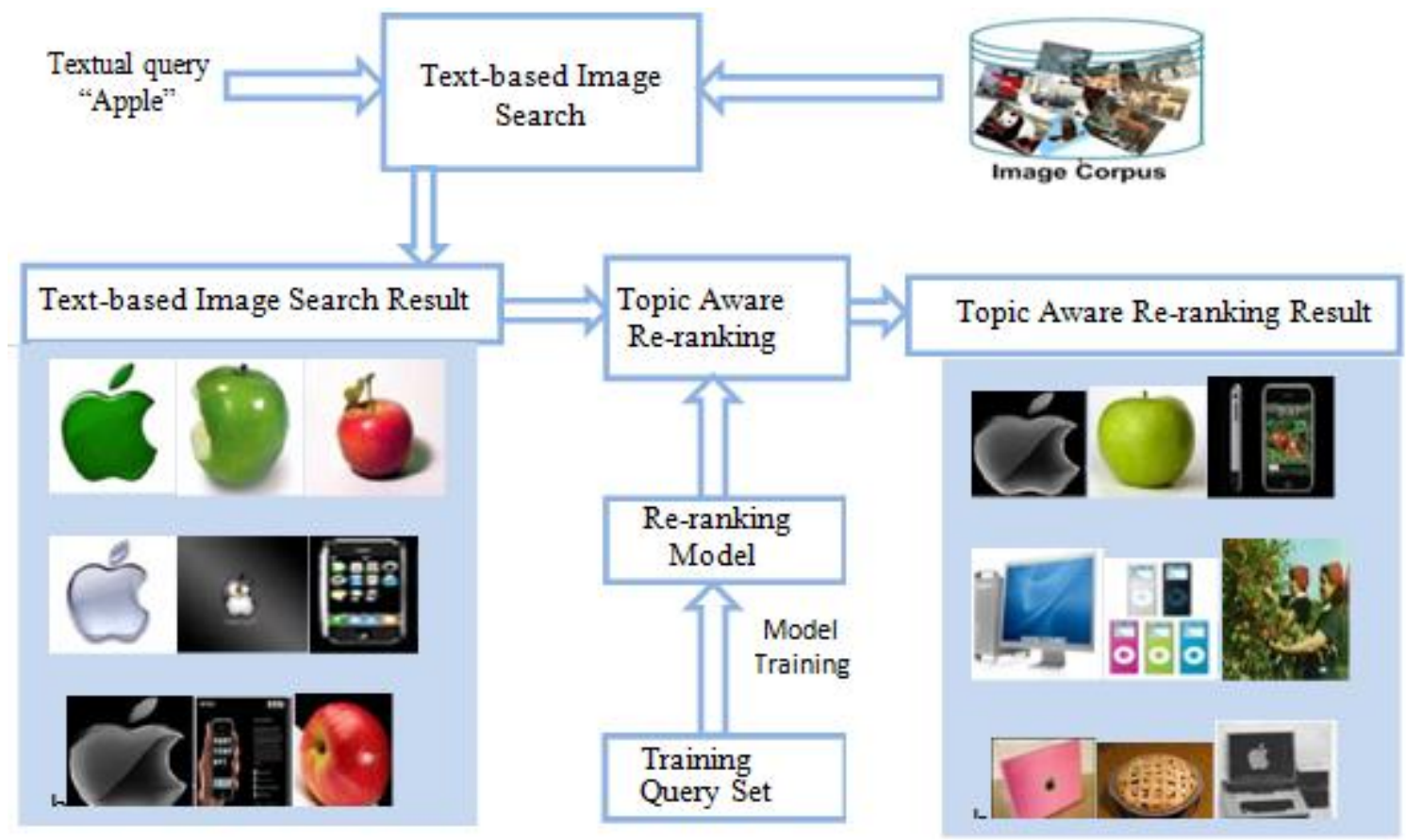

Figure 2: Proposed system architecture 


\section{International Journal of Science and Research (IJSR) \\ ISSN (Online): 2319-7064 \\ Index Copernicus Value (2013): 6.14 | Impact Factor (2014): 5.611}

This estimation thinks seriously about topic significance, and is appropriate for managing various leveled topics.

\section{Applications}

Image search has becoming a cornerstone of many commercial search engines. Some of the applications constraints of the Image Search Re-ranking are as follows:

\section{- Web Image Search Engine}

Bing, Google and Yahoo! are executed by indexing and searching the printed data connected with images, for example, image record names, encompassing writings, all inclusive asset locator, et cetera. In spite of the fact that content based image search is powerful for huge scale image accumulations, it experiences the downside that printed data can't extensively and considerably portray the rich substance of images. As an outcome, some insignificant images are seen in the search results. To handle the troubles in content based image search, Relevance Preserving Projection for Web image Search Engine Re-ranking with hierarchical topic awareness is best way.

\section{- Medical Science}

In this field through medical report images, we can compare various types of diseases with Web image Search re-ranking about the most topic awareness in hierarchical structure. So that the identification of the major problem will be search is in easy way manner.

\section{- Botanical Aspects}

Plant Species identification using leaf image retrieval is best way consideration for the Relevance Preserving Projection for image Search Engine Re-ranking with hierarchical topic awareness. In this area shape feature extraction techniques have to be implemented successfully for sick of image retrieval.

\section{- Remote Sensing Area}

Remote sensing imagery needs to be converted into tangible information which can be utilized in conjunction with other dataset, often within widely used Geographic Information Systems (GIS). The ranking system in these area mainly focus on two aspect of the images, focal pixel size of the object image and major distances from capturing angel.

\section{Conclusion}

In this paper, we proposed enhanced reranking system, TARerank, used to refine text based image search reranking. TARerank takes topic into consideration and likewise takes in an optimizing so as to rerank model a basis identified with reranking execution as far as both relevance and differences in one stage at the same time. To better model the various leveled theme structure of search results and portray the relevance and assorted qualities in one basis flawlessly, NCTC is proposed to measure the progressive TC. Contrasted and the two-stage improvement in other expanded reranking routines, TARerank can accomplish the joint ideal of enhancing relevance and differing qualities. Additionally, the learning methodology can overcome any issues between low-level visual component assorted qualities and abnormal state semantic theme differing qualities to some degree. These two favorable circumstances guarantee the prevalence of TARerank. By leading broad tests on a Web image dataset, we have exhibited the adequacy of the proposed system. Moreover, we find that both the relevance and TC are enhanced in proposed TARerank. This method is a promising new worldview for Web image search reranking.

Future work will investigate some extra goals. It include semantic data in TC highlight development and further overcome any issues between visual differences and subject assorted qualities. At present, the NCTC can just manage two relevance levels. In this way, creating multilevel relevance in the NCTC and TARerank is a heading for future research.

\section{References}

[1] W. Cao, N. Liu, Q. Kong, and H. Feng, "Content-based image retrieval using high-dimensional information geometry, "Sci. China Tech. Sci., vol. 57, no.7,pp. 111, 2014"

[2] B. Geng, L. Yang, C. Xu, and X. Hua, "Content-aware ranking for visual search", in Proc. IEEE Int. Conf. Comput. Vis. Pattern Recognit, 2010, pp. 3400-3407.

[3] R. Fisher, "The use of multiple measurements in taxonomic problems." Anuals of Eugenics, vol. 7, no.2, 179-188,1936.

[4] X. He, S. Yan, Y. Hu, P. Niyogi, and H. Zhang, "Face recognition using Laplacianfaces," IEEE Trans. Pattern Anal. Mach. Intell., vol. 27, no. 3, pp. 328 340, 2005.

[5] G. Hua, M. Brown, and S. Winder, "Discriminant embedding for local image descriptors," in Proc. IEEE Int. Conf. on Comput. Vis., 2007, 1-8.

[6] W. Hsu, L. Kennedy, and S. Chang, "Video search reranking through random walk over document-level context graph," in Proc. ACM Int.Conf. Multimedia, 2007, pp. 971-980.

[7] I. Jolliffe, Principal Component Analysis, SpringerVerlag, 1986.

[8] X. Li. and Y. Pang, "Deterministic Column-Based Matrix Decomposition," IEEE Trans knowl. Data Eng., vol. 22, no.1,pp.145-149,2010.

[9] Y. Yang, W. Hsu, and H. Chen, "Online reranking via ordinal informative concepts for context fusion in concept detection and video search", IEEE Trans. Circuits Syst. Video Technol., vol. 19, no. 12, pp.1880 1890, 2009.

[10] Y. Liu, T. Mei, X. Hua, J. Tang, X. Wu, and S. Li, "Learning to video search rerank via pseudo preference feedback," in Proc. IEEE Int Conf. Multimedia and Expo, 2008, pp. 207-210.

[11]T. Mei, Y. Rui, S. Li, and Q. Tian, "Multimedia search reranking: a literature survey," ACM Computing Surveys, vol. 46, no. 3, pp. 1-38, 2014.

[12] Y. Pang, X. Jiang, X. Li, and J. Pan, "Efficient object Detection by Prediction in 3D space," signal Proc., vol. 112, pp. 64-73, 2015. 
[13] Y. Pang, X. Li, and Y. Yuan, "Robust Tensor Analysis with L1- Norm." IEEE Trans. Circuits syst. Video Technol., vol. 20, no. 2, pp. 172-178,2010.

[14] J. Tang, L. Shao, and X. Li, "Efficient dictionary learning for visual categorization," Comput. Vis. Image Underst, vol. 124, pp. 91-98, 2014.

[15] J. Tešić, A. Natsev, L. Xie, and J. Smith, "Data modeling strategies for imbalanced learning in visual search," in Proc. IEEE Int. Conf.Multimedia and Expo, 2007, pp. 1990-1993.

[16] X. Tian, L. Yang, J. Wang, X. Wu, and X.-S. Hua, "Bayesian visual reranking," IEEE Trans. Multimedia, vol. 13, no. 4, pp. 639-652, Aug. 2011.

[17] M. Wang, H. Li, D. Tao, K. Lu, and X. Wu, "Multimodal graph-based reranking for web image search," IEEE Trans. Image Process., vol. 21, no. 11, pp. 4649-4661, 2012.

[18] D. Xu, S. Yan, D. Tao, S. Lin and H. Zhang, "Marginal fisher analysis and its variants for human gait recognition and content based image retrieval," IEEE Trans. Image Process., vol. 16, no. 11, pp. 2811-2821, 2007.

[19] R. H. van Leuken, L. G. Pueyo, X. Olivares, and R. van Zwol, "Visual diversification of image search results," in Proc. Int. World Wide Web Conf. (WWW), Madrid, Spain, 2009, pp. 341-350.

[20] J. Yu, Y. Rui, and D. Tao, "Click Prediction for Web Image Reranking Using Multimodal Sparse Coding." IEEE Trans. Image Process., vol. 23, no.5, pp. 20192032, 2014.

[21] J. G. Carbonell and J. Goldstein, "The use of MMR, diversity-based reranking for reordering documents and producing summaries," in Proc.ACM SIGIR Spec. Interest Group Inf. Retrieval, Melbourne, VIC, Australia, 1998, pp. 335-336.

[22] Y. Zhang, X. Yang, and T. Mei. "Image Search Reranking with Query-dependent Click-based Relevance Feedback," IEEE Trans. Image Process., vol. 23, no. 10, pp. 4448-4459, 2014.

[23] Junjie Cai, Zheng-Jun Zha, Meng Wang, Shiliang Zhang, and Qi Tian , “An Attribute-assisted Reranking Model for Web Image Search," IEEE TRANSACTIONS ON IMAGE PROCESSING, VOL. X, NO. 1057-7149 (c) 2013 IEEE. 